\title{
Review
}

\section{Recent Progress on the Development of Tomographic Models}

\author{
X. George XU \\ (Received on June 20 2006) \\ (Accepted on June 20, 2006)
}

\begin{abstract}
We are at a critical time in the history of radiation protection dosimetry. The paradigm shift away from the more-than-30years-old stylized human models is imminent with the development of 30 voxel-based tomographic models in recent years that are based on medical images. Recently, researchers gathered at an international conference to share the latest work and to establish collaborations. This invited review paper introduces various types of modelling and summarizes the latest developments including a Consortium on Computational Human Phantoms (CCHP) that is aimed at facilitating intercomparison and sharing of data for radiation protection purposes.
\end{abstract}

KEY WORDS: tomographic model, dosimetry, medical imaging, Monte Carlo, medical images.

\section{INTRODUCTION}

One of the most active areas of research and development in radiation protection dosimetry has been the advanced methods in designing computational models (or phantoms) that represent radiation workers. Such computational models are used to derive many of the existing dosimetric parameters. In external dosimetry, for example, fluence-to-dose-equivalent conversion factors are widely used for dosimeter calibration and shielding design. 1) On the other hand, the specific absorbed fractions (SAFs) or the specific effective energies (SEEs) are used to assess target organ doses due to internally deposited radionuclides in the source organ(s) following an accidental intake or a nuclear medicine procedure. ${ }^{2,3)}$ These parameters are determined using full-body human anatomical models containing radiosensitive organs. The list of these organs as well as the associated organ weighting factors often change as a result of recommendations by the International Commission on Radiological Protection (ICRP) ${ }^{2,3)}$. These worker anatomical models are used to accurately 3-dimensional radiation transport and energy deposition using the Monte Carlo methods. It is apparent that the accuracy of these dosimetric quantities (and others derived from them) depends upon how good the worker models are designed. During the past decade, a paradigm change in the way the worker's anatomy is modelled has occurred with the emergency of the so-called "voxel models." On-going research projects in the United States and elsewhere have led to new approaches that may further shape the radiation protection dosimetry in the future. This review summarizes the latest efforts and future

* Nuclear Engineering and Engineering Physics, School of Engineering, Rensselaer Polytechnic Institute, Room 1-11, NES Building, Tibbits Ave., Troy, New York 12180, USA.

E-mail: xug2@rpi.edu. directions in the development of computational worker models for radiation protection purposes.

\section{EXISTING AND EMERGING MODELS}

To date, three types of human body model have been developed for radiation protection dosimetry: 1) Equation-based stylized models, where organs are delineated by a combination of simple surface equations, 2) Image-based tomographic models, in which organs are defined from segmented and labeled volume elements (voxels) from tomographic images of real individuals, and 3) Hybrid equation-voxel models that mathematically describe organ boundaries from definitions extracted from voxel data. These models are described in details below.

\section{Stylized Models}

Early models for radiation protection dosimetry were mostly homogeneous slabs, cylinders, and spheres. The first heterogeneous anthropomorphic model was devised at Oak Ridge National Laboratory (ORNL) for the Medical Internal Radiation Dose (MIRD) Committee of The Society of Nuclear Medicine. ${ }^{4,5)}$ This stylized model, also known as the MIRD Model or Mathematical Model, was based on the definition of "Reference Man" for radiation protection purposes. ${ }^{6)}$ The Reference Man was a 20-30 year old Caucasian, weighing $70 \mathrm{~kg}$ and $170 \mathrm{~cm}$ in height. The original MIRD stylized model was analytically described in three principal sections: an elliptical cylinder representing the arm, torso, and hips; a truncated elliptical cone representing the legs and feet; and an elliptical cylinder representing the head and neck, as shown in the Fig. 1. Later improvements at ORNL have led to a "family" of models having both genders at various ages. 7) Using the original descriptions, others have developed modified versions such as those known as the "Adam" and "Eva". ${ }^{8)}$ A more recent revision to the head and brain model contained sub- 


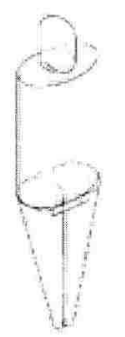

(a)

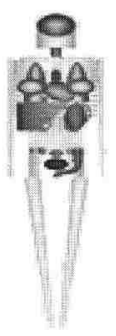

(b)

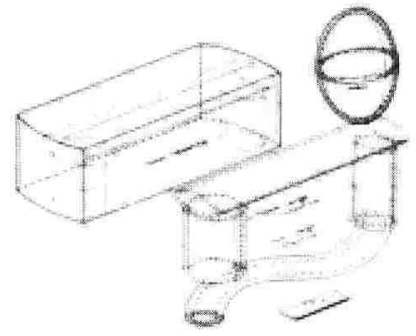

(c)

Fig 1. Stylized adult male models showing (a) exterior view of the adult male, (b) skeleton and internal organs of the adult male/female, (c) surface equations representing stomach and GI tract.

regions of the brain. ${ }^{9)}$ For many years, these models have served practically as the de facto "standard" to the radiation protection dosimetry community. ${ }^{10.11)}$.

The stylized mathematical descriptions of the organs were formulated based on descriptive and schematic materials from general anatomy references. More than 40 organs and tissues were specified, with basically three media of distinct densities: bone, soft tissue, and lung. The goal of this approach was to make the equations simple, thus accommodating the computing abilities in 1970s (ICRP 1987; ICRP 1996). For example, the stomach wall (the geometry looked like an American football in Fig. 1c) is defined as the volume between two concentric ellipsoids ${ }^{7)}$ :

$$
\begin{aligned}
& {\left[\frac{x-x_{0}}{a}\right)^{2}+\left(\frac{y-y_{0}}{b}\right)^{2}+\left(\frac{z-z_{0}}{c}\right)^{2} \leq 1} \\
& \text { and }\left[\frac{x-x_{0}}{a-d}\right)^{2}+\left(\frac{y-y_{0}}{b-d}\right)^{2}+\left(\frac{z-z_{0}}{c-d}\right)^{2} \geq 1 .
\end{aligned}
$$

It is clear, however, that the human anatomy is too complex to be realistically modeled with a limited set of equations. As such, many anatomical details in the stylized models had to be compromised. Although the organ shapes and locations can be further adjusted, the use of quadrics means that they will remain simplified and crude. For instance, the skeleton in the stylized adult male model does not resemble a human. In the meantime, computers have become much more powerful and many realized that it was technically no longer necessary to limit the geometry representation to overly simplified shapes.

\section{Tomographic Models}

During the past two decades, a new class of computational models has emerged with the advent of $3 D$ tomographic imaging and powerful computers. 3-D medical imaging modalities, such as Computed Tomography (CT) and Magnetic Resonance Imaging (MRI) allow us to easily visualize the internal structures of the body and to store the images in versatile digital formats. Tomographic images reveal patient internal structures accurately, but time-consuming segmentation and classification are necessary to transform the images into useful geometry descriptions for radiation transport calculations in a Monte Carlo code. Tomographic models contain large arrays of voxels that are identified in terms of tissue type (soft tissue, hard bone, air, etc) and unique organ identification (lungs, liver, skin, etc). Since the voxels are the basic elements of this type of models, many called this type of models "voxel models" to indicate the different approach from the older stylized, mathematical models. Table 1 summarizes more than 30 whole-body tomographic models that have been reported in the literature. ${ }^{12} \sim 28$ ) These models contain organs that are segmented and classified, thus are ready for implementation into a Monte Carlo code. Table 1 is an updated version of the table from a recent review paper. ${ }^{29}$ \}

To illustrate the tomographic models, Fig. 2 shows the VIPMan model developed at Rensselaer. ${ }^{23)}$ This model was based

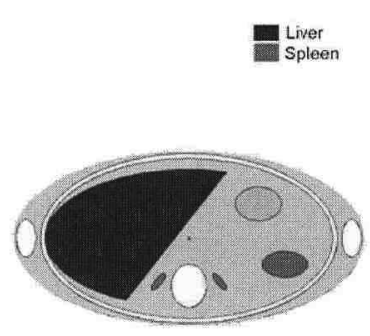

MIRD Stylized Model

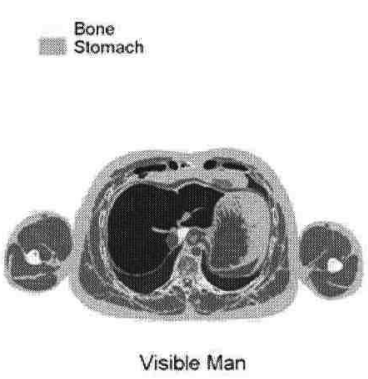

(a)

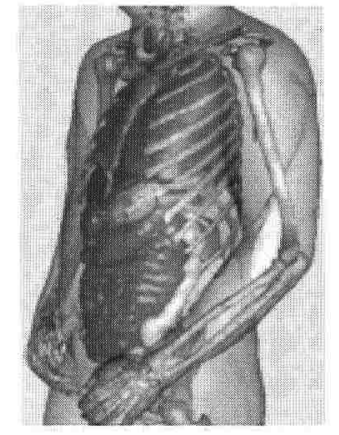

(b)

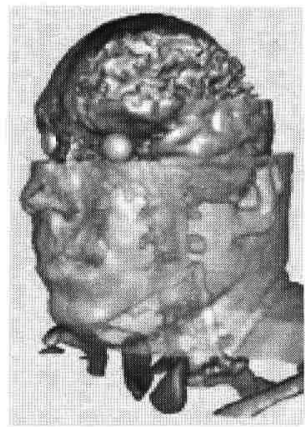

(c)

Fig 2. (a) The VIP-Man model has obviously different anatomical representation from the stylized model. (b) VIP-Man in 3-D views showing details of internal organs and the skeleton; (c) Details of the head and brain. 
Table 1 Existing tomographic and hybrid human models.

\begin{tabular}{|c|c|c|c|c|c|c|}
\hline Developer & Name & Images & Race & Age and sex & Subject & Comment \\
\hline $\begin{array}{l}\text { Flinders University, } \\
\text { Australia [12] }\end{array}$ & ADELAIDE & $\mathrm{CT}$ & Caucasian & 14-year-old female & Patient & Torso \\
\hline $\begin{array}{l}\text { Federal University of } \\
\text { Pernambuco,Brazil [13] }\end{array}$ & MAX & $\mathrm{CT}$ & Caucasian & Adult male & Patient & Added arms and legs to the Zubal model. \\
\hline $\begin{array}{l}\text { Federal University of } \\
\text { Pernambuco, Brazil [14] }\end{array}$ & FAX & CT & Caucasian & Adult female & Patient & \\
\hline GSF, Germany [15] & Baby & $\mathrm{CT}$ & Caucasian & 8-week-old female & Cadaver & \\
\hline GSF, Germany [15] & Child & $\mathrm{CT}$ & Caucasian & 7-year-old female & Leukemia patient & Small for age(5 to 7-year-old) \\
\hline GSF, Germany [15] & Donna & $\mathrm{CT}$ & Caucasian & 40-year-old female & Patient & \\
\hline GSF, Germany [15] & Frank & $\mathrm{CT}$ & Caucasian & 48-year-old male & Patient & Head and torso \\
\hline GSF, Germany [15] & Golem & $\mathrm{CT}$ & Caucasian & 38-year-old male & Leukemia patient & \\
\hline GSF,Germany [15] & Helga & $\mathrm{CT}$ & Caucasian & 26-year-old female & Patient & Legs absent below mid-thigh \\
\hline GSF, Germany [15] & Irene & $\mathrm{C} \Gamma$ & Caucasian & 32-year-old female & Patient & \\
\hline GSF, Germany [15] & Visible Man & $\mathrm{CT}$ & Caucasian & 39-year-old male & Cadaver(VHP) & No arms \\
\hline NIICT, Japan [16] & Nagaoka man & MRI & Japanese & 22-year-old male & Volunteer & \\
\hline NIICT, Japan [16] & Nagaoka woman & MRI & Japanese & 22-year-old female & Volunteer & \\
\hline JAERI, Japan [17] & Otoko & $\mathrm{CT}$ & Japanese & Adult male & Healthy Volunteer & \\
\hline JAERI, Japan [17] & Otoko & $\mathrm{CT}$ & Japanese & Adult female & Healthy Volunteer & \\
\hline JAERI, Japan [18] & JM & $\mathrm{CT}$ & Japanese & 55-year-old,male & Healthy Volunteer & \\
\hline JAERI, Japan [19] & JF & CT & Japanese & Adult female & Healthy Volunteer & \\
\hline $\begin{array}{l}\text { Johns Hopkins } \\
\text { University, USA [20] }\end{array}$ & NCAT & CT & Caucasian & 39-year-old male & Cadaver(VHP) & No arms, Motion-simulating $\backslash$ \\
\hline $\begin{array}{l}\text { Hanyang University, } \\
\text { Korea [21] }\end{array}$ & KORMAN & MRI & Korean & 28-year-old male & & \\
\hline NRPB, UK [22] & NORMAN & MRI & Caucasian & Adult male & & Only 10 ribs \\
\hline RPI, USA [23] & Pregnant woman & CT & & 30 weeks pregnant & & Part torso \\
\hline RPI, USA [24] & $\begin{array}{l}\text { VIP-Man } \\
\text { photos }\end{array}$ & Color & Caucasian & 39-year-old male & Cadaver (VHP) & One testicle only \\
\hline RPI, USA [25] & $\begin{array}{l}\text { VIP-Man 4D } \\
\text { photos }\end{array}$ & Color & Caucasian & 39-year-old male & Cadaver (VHP) & Only chest; Motion-simulating \\
\hline UF, USA [26] & UF 2 Month & $\mathrm{CT}$ & Caucasian & 6-month-old male & Cadaver & \\
\hline UF, USA [26] & UF Newborn & $\mathrm{CT}$ & Caucasian & 6-day-old female & Cadaver & \\
\hline UF, USA [27] & UF 9 Month & CT & & 9-month-old male & Patient & Head and torso \\
\hline UF, USA [27] & UF 4-y & CT & & 4-year female & \begin{tabular}{|l|} 
Patient \\
\end{tabular} & \\
\hline UF, USA [27] & UF 8-y & $\mathrm{CT}$ & & 8-year female & Patient & \\
\hline UF, USA [27] & UF 11-y & $\mathrm{CT}$ & & 11-year male & Patient & \\
\hline UF, USA [27] & UF 14-y & $\mathrm{CT}$ & & 14-year male & \begin{tabular}{|l|} 
Patient \\
\end{tabular} & \\
\hline Yale, USA [28] & Zubal & $\mathrm{CT}$ & Caucasian & Adult male & Patient & Head and torso \\
\hline
\end{tabular}

cross sectional color photographic images for a 39 year old male cadaver from the Visible Human Project (VHP). ${ }^{30} \sim 32$ ) The transversal anatomical images were obtained by photographing the top surface of the body block after removal of (by shaving) each successive millimeter ( 0.3 millimeter for the female cadaver) by a cryomacrotome. Each anatomical photograph represents a $1-\mathrm{mm}$ thick slice for the male cadaver. These data covered the entire body with about 4 billion voxels, each having a small size of $0.33 \mathrm{~mm} \times 0.33 \mathrm{~mm} \times 1 \mathrm{~mm}$ for the male. The small voxel size in this model allowed many small organs and tissues to be represented or represented more accurately than some of the other models. In addition to the color photos, CT and MR images were also taken during the Visible Human Project. The VHP CT images have been used by several groups to develop tomographic models. ${ }^{15,20)}$ The availability of identical cross sectional color images and CT images from the VHP allow unique dosimetric investigations. For example, the red bone marrow distribution in the color images can be used to benchmark methods of segmenting this tissue from the CT. The finalized VIP-Man model shows a heavy body weight of $103 \mathrm{~kg}$ (due to a relatively large portion of fatty tissues). However, most organs have similar masses as those of the Reference Man. If necessary, the size and shape of the organs in the VIP-Man model can be individually adjusted to match closely with the Reference Man. To date, the model has been coupled with various Monte Carlo codes, for radiation transport studies and organ dose calculations involving photons, electrons, neutron, and protons. For more information on the VIP-Man model, please visit RRMDG.rpi.edu.

\section{Hybrid Models}

This is a very new type of model that was primarily developed to address problems associated with cardiac and respiratory motions during medical imaging and radiation therapy. Organ motions involve tissue deformation - a process that is difficult to handle using voxel-based data format. The new approach combines voxel data with advanced surface descriptions, such as the Non-uniform rational b-splines (NURBS), ${ }^{33)}$ to design hybrid models where organ shapes are more realistic than stylized models, while maintaining the flexibility for anatomical variations and organ deformation. In this type of geometry-based models, one can adjust the NURBS surface control points of an organ to the desired shape and volume using patient-specific images and deformable image registration. Examples of such hybrid models include the 4D 
NURBS-based Cardiac-Torso (NCAT) model by Segars from Johns Hopkins using the VHP CT images ${ }^{20)}$ and those by Xu and Shi from Rensselaer using the VHP anatomical color images, respectively. ${ }^{25)}$

\section{RECENT DEVELOPMENTS}

Since 1998, the ICRP's Task Group on Dose Calculations (DOCAL) and the SNM's MIRD committee have been evaluating new dosimetry data from these tomographic models. In particular, the DOCAL, which is administered by the ICRP Committee 2, has been developing international guidelines on the use of voxel-based models. In an ICRP Annual Report in $2002^{34)}$ a paradigm shift in the way the human body is modeled for radiation protection dosimetry was predicted: “An important issue for Committee 2 is the substitution of an anatomically realistic voxel phantom, obtained digitally in magnetic resonance tomography and/or computed tomography, for the MIRD phantom which is a mathematical representation of a human body." The ICRP has made the decision to adopt a revised version of the Golem model that was developed at GSF as the "standard" tomographic model of an adult male worker. The standard female model is based on Laura, also developed at GSF. Some of the last-minute efforts at GSF include final adjustment of the details in light of new data published in ICRP 89 and the draft ICRP 2005 Recommendations. ${ }^{35,36)}$ It is expected that the ICRP will release the guidelines and finalized ICRP standard models in 2006.

The radiation dosimetry research community had been mostly uninformed of the latest development and most tomographic model researchers had little chance to exchange ideas. Although more than 30 tomographic models have been developed, our efforts to date have been largely isolated, and collaborations at any level were sparse. As a result, resources were not utilized efficiently. It is still unknown how much the radiation protection dosimetry has been improved due to these tomographic models. In one attempt to address some of the issues, a special conference session "Tomographic Models for Radiation Protection Dosimetry" was organized during the Monte Carlo 2005 Topical Meeting in Chattanooga, TN, USA, April 17-21, 2005. Ten speakers from different countries were invited to report the latest research and development. During the conference, further discussions on collaboration led to the official formation of the Consortium on Computational Human Phantoms (CCHP) (www.virtualphantoms.org). The Founding members of The Consortium of Computational Human Phantoms (CCHP):

- X. George Xu, Rensselaer Polytechnic Institute (Contact Person, xug2@rpi.edu), USA;

- Wesley E. Bolch, University of Florida, USA;

- Loic de Carlan, IRSN -Institute of Nuclear Safety and Radiation Protection, France;

- Martin Caon, Flinders University, Australia;

- Keith F. Eckerman, Oak Ridge National Laboratory, USA;

- Rickard Kramer, Federal University of Pernambuco, Brazil;
- Choonsik Lee, Hanyang University, Korea;

- Tomoaki Nagaoka, Kitasato University Graduate School of Medical Sciences, Japan;

- Lawrence S. Pinsky, University of Houston, USA;

- Kimiaki Saito, Japan Atomic Energy Research Institute, Japan;

- William Segars, Johns Hopkins University, USA;

- Michael G. Stabin, Vanderbilt University, USA;

- Maria Zankl, GSF - National Research Center for Environment and Health, Germany;

- George Zubal, Yale University, USA.

The CCHP website has received several hundreds of visits since October 2005 and the membership has been steadily increasing. It is expected that, in the future, the CCHP will become a center of resources for human modeling for radiation protection dosimetry. One of the ultimate goals of this consortium is to compile and share the phantoms as well as software tools. Proper intellectual protection measures will have to be developed among the developers. Under the leadership of the funding members, a series of activities will be planned including conferences, workshops, research proposals, and publications. The 2005 meeting in Chattanooga, TN was one of the first events to come. Presentations at this and other meetings are available to download from the CCHP's website. If you are interested in becoming a member of the CCHP, please contact Dr. X. George Xu [xug2@rpi.edu].

\section{SUMMARY}

We are at a critical time in the history of radiation protection dosimetry. The research community and the ICRP are decidedly shifting away from the stylized models that have dominated since 1970 s to tomographic models that are more realistic in anatomy and physiology. To date, more than 30 models have already been reported in the literature. The ICRP has made the decision to adopt two adult male and female tomographic models as the "standard" tomographic models.

Voxel-based geometry lacks the flexibility for organ adjustment and is hardly the ideal format for human body modelling. Despite the fact that many tomographic models have been developed, the improvements to health physics dosimetry due to these tomographic models may be insignificant for most common radiation exposures in health physics as long as the "Reference Man" concept is continued. ${ }^{37)}$ However, the adoption of medical images has opened the door for realistic and person-specific dosimetry. Although additional new whole-body tomographic models will likely continue to be developed in the near future, it is the author's opinion that the community would benefit from new models that fill certain ethnic and/or age groups, contain new organs or provide additional features such as motion simulations. Another area that has been lacking is the intercomparison among existing models, especially involving the new ICRP standard models to be released in the near future. Future research will also likely develop advanced and user-friendly computer graphic tools to 
facilitate the adjustment of the body and organs to any desired patient-specific shape and size. New capabilities in simulating multiple particle transport in complex voxel geometries will be highly desirable because some of the most widely used Monte Carlo codes (such as MCNP code) do not efficiently handle large amounts of voxels or moving targets. Last but not least, there will be a need to create physical phantoms to benchmark the dosimetry data obtained from tomographic models. The combination of medical imaging and 3D rapid prototyping for CAD-based phantom fabrication is an area that may deserve special attention in future research. Research activities beyond 5-10 years from today will be largely driven by applications in medical imaging and radiotherapy where accurate patient-specific modelling is critical. One should not be surprised if future technologies will again bring opportunities for new ways of human modelling (in the same way that the $3 \mathrm{D}$ imaging made the tomographic models possible). After all, what have happened in radiation protection dosimetry in the past decade or so are merely the result of a larger scientific movement called "Virtual Human" or "Digital Human" that is aimed at creating an ultimate biomedical framework that addresses various environmental stimuli including ionizing radiation with increasing level of details.

\section{Acknowledgements}

The original VIP-Man model was developed from the National Library Medicine's Visible Human data through doctoral research projects involving two students at Rensselaer, TC Chao and A Bozkurt. The preliminary 4D motion-simulating VIP-Man model was developed by a doctoral student at Rensselaer, CY Shi. These research activities were funded by the U.S. National Science Foundation/Biomedical Engineering Program under a Faculty Early Career Development Grant Award (\#BES-9875532) and U.S. National of Institutes of Health (1 R03 LM007964-01, 1 R42 CA115122-01 and 1R01CA116743).

\section{REFERENCES}

1. International Commission on Radiation Units and Measurements; Conversion coefficients for use in radiological protection against external radiation, ICRU Report 57 (1998).

2. International Commission on Radiological Protection; Recommendations of the International Commission on Radiological Protection, ICRP Publication 26 (1977).

3. International Commission on Radiological Protection; Recommendations of the International Commission on Radiological Protection, ICRP Publication 60 (1990).

4. W. S. SNYder, M. R. Ford, G. G. WARNER and H. L. Fisher Jr.; Estimates of absorbed fractions for monoenergetic photon source uniformly distributed in various organs of a heterogeneous phantom, Medical Internal Radiation Dose Committee (MIRD) Pamphlet No.5, Supplement No. 3, J. Nucl. Med., Society of Nuclear Medicine 10, New York (1969).

5. W.S. SNYDER, M.R. Ford, G.G. WARNER and H.L. FiSHER Jr.; Estimates of absorbed fractions for monoenergetic photon source uniformly distributed in various organs of a heterogeneous phantom. Medical Internal Radiation Dose Committee (MIRD) Pamphlet No. 5, Revised (1978).

6. International Commission on Radiological Protection; Report of the task group on Reference Man, ICRP Publication 23, (1975).

7. M CRISTY and K.F. ECKERMAN; Specific absorbed fractions of energy at various ages from internal photon sources. Report No. ORNL/TM-8381/V1 Oak Ridge, TN: Oak Ridge National Laboratory (1987).

8. R. Kramer, M. ZanKL, G. Williams and G. DreXler; The calculation of dose from external photon exposures using reference human phantoms and Monte-Carlo methods, Part 1: The male (ADAM) and female (EVA) adult mathematical phantoms, GSF Bericht S-885, December (1982).

9. L.G. Bouchet, W.E. Bolch, D.A. Weber, H.L. Atkins, and J.W. Sr. Poston; A revised dosimetric model of the adult head and brain. J. Nucl Med. 37 1226-1236 (1996).

10. International Commission on Radiation Units and Measurements; Phantoms and computational models in therapy, diagnosis and protection, ICRU Report 48, (1992).

11. International Commission on Radiological Protection; Conversion coefficients for use in radiological protection, against external radiation, ICRP Publication 74, (1996).

12. M. CaOn, G. BibBo and J. Pattison; An EGS4-ready tomographic computational model of a 14-year-old female torso for calculating organ doses from CT examinations, Phys. Med. Biol., 44 (9), 2213-2225 (1999).

13. R. Kramer, J.W. Vieira, H.J. Khoury, F.R.A. Lima and D. FUELLE; All about MAX: a male adult voxel phantom for Monte Carlo calculations in radiation protection dosimetry, Phys. Med. Biol. 48, 1239-1262 (2003).

14. R. Kramer, J.W. Vieira, H.J. Khoury, F.R.A. Lima, E.C.M. LOUREIRO, V.J.M. LIMA and G. HofF; All about FAX: a Female Adult voXel phantom for Monte Carlo calculation in radiation protection dosimetry, Phys. Med. Biol., 49, 5203-5216 (2004).

15. N. P-H, M. ZANKL, U. Fill and D. Regulla; The GSF family of voxel phantoms. Phys. Med. Biol., 47, 89-106 (2002).

16. T. Nagaoka, S. Watanabe, K. Sakurai, E. Kunieda, S. WATANABE, M. TAKi and Y. YamanaKa; Development of realistic high-resolution whole-body voxel models of Japanese adult males and females of average height and weight, and application of models to radio-frequency electromagnetic-field dosimetry. Phys. Med. Biol., 49 (1), $1-15$ (2004).

17. K. Saito, A. Wittmann, S. Koga, Y. Ida, J. Kamei, J. FUNABIKI and M. Zankl; Construction of a computed tomographic phantom for a Japanese male adult and dose calculation system, Radiat. Environ. Biophys., 40, 69-76 (2001).

18. K. Sato, H. Noguchi, Y.S. Emoto Koga and K. Saito; Japanese adult male voxel phantom constructed on the basis of CT-images, Radiat. Protect. Dosim. (submitted).

19. K. Sato, H. Noguchi, Y.S. Emoto Koga and K. Saito; Construction of a Japanese adult female voxel phantom 
for internal dosimetry, Radiat. Environ. Biophys., (submitted).

20. W.P. Segars; Development and application of the new dynamic NURBS-based cardiac-torso (NCAT) phantom, Dissertation in Biomedical Engineering, University of North Carolina, Chapel Hill, NC (2001).

21. C. LeE, J. LeE and C. LeE; Korean adult male voxel model KORMAN segmented from magnetic resonance images. Med. Phys., 31(5), 1017-1022 (2004).

22. D.G. Jones; A realistic anthropomorphic phantom for calculating organ doses arising from external photon irradiation. Radiation Protection Dosimetry. Radiat. Prot. Dosim., 77 (1), 21-29 (1994).

23. C.Y. SHI and X.G XU; Development of a 30-week-pregnant female tomographic model from CT-images for Monte Carlo organ dose calculations, Med. Phys., 31(9), $2491-2497$ (2004).

24. X.G. Xu, T.C. Chao and A. Bozkurt; VIP-Man: an image-based whole-body adult male model constructed from color photographs of the visible human project for multi-particle Monte Carlo calculations, Health. Phys., 78 (5), 476-486 (2000).

25. X.G. XU and C.Y. SHI; Preliminary Development Of A 4d Anatomical Model For Monte Carlo Simulations. Monte Carlo 2005 Topical Meeting. The Monte Carlo Method: Versatility Unbounded In A Dynamic Computing World, Chattanooga, TN, April 17-21 (2005).

26. J.C. NiPPER, J.L. WiLliams and W.E. BolCH; Creation of two tomographic voxel models of pediatric patients in the first year of life, Phys. Med. Biol., 47, 3143-3164 (2002).

27. C. LeE, J.L. Williams, C. LeE, W.E. Bolch; The UF series of tomographic computational phantoms of pediatric patients., Med. Phys. 32 (12) 3537-3548 (2005).
28. I.G. Zubal, C.R. Harrel, E.O. Smith, Z. Rattner, G. GINDI, and P.B. Hoffer; Computerized Three-dimensional Segmented Human Anatomy, Med. Phys., 21(2), 299302 (1994).

29. M.CAON; Voxel-based computational models of real human anatomy: a review, Radiat Environ Biophys., 42 $229-235$ (2004).

30 M.J. ACKERMAN; Accessing the visible human project. Dlib Magazine: The Magazine of the Digital Library Forum (1995).

31. National Library of Medicine (NLM), U.S. Board of Regents. Electronic imaging: Report of the Board of Regents, NIH Publication 90-2197 (1990).

32. V.M. Spitzer and D.G. Whitlock; "Atlas of the Visible Human Male" (1998). Jones and Bartlett Publishers.

33. L. Piegl, W. Tiller; "The Nurbs Book" (1997), Springer-Verlag, New York.

34. ICRP Report. http://www.icrp.org/docs/2002_Ann_Rep_ 52_429_03.pdf. Last accessed April 24 (2006).

35. International Commission on Radiological Protection; Basic anatomical and physiological data for use in radiological protection: reference values. A report of age- and gender-related differences in the anatomical and physiological characteristics of reference individuals, Pub. 89, Ann. ICRP, 32 (3-4), 5-265 (2002).

36. ICRP. 2005 Recommendations of The International Commission On Radiological Protection http://www.icrp. org/docs/2005_recs_CONSULTATION_Draft1a.pdf. Last accessed April (2006).

37. X.G. Xu, T.C Chao and A. Bozkurt; Comparison of Effective Doses from Various Monoenergetic Particle Based On the Stylized and the VIP-Man Tomographic Models. Rad Prot. Dosimetry, 115 (1-4), 530-535 (2005). 